\title{
Investigation of the relationship between atopy and psoriasis
}

\author{
Pegah Hosseini ${ }^{1}$, Maryam Khoshkhui ${ }^{2}$, Reza Farid Hosseini' ${ }^{2}$ Hamid Ahanchian ${ }^{3}$, Yalda Ravanshad ${ }^{4}$, Pouran Layegh ${ }^{5}$, \\ Behnoosh Bakhshoudeh ${ }^{1}$, Nazila Ariaee ${ }^{2}$
}

${ }^{1}$ Student Research Committee, Mashhad University of Medical Sciences, Mashhad, Iran ${ }^{2}$ Allergy Research Center, Mashhad University of Medical sciences, Mashhad, Iran

IInflammation and Inflammatory Diseases Research Center, Mashhad University of Medical Sciences, Mashhad, Iran ${ }^{4}$ Clinical Research Development Units, Mashhad University of Medical Sciences, Mashhad, Iran

${ }^{5}$ Cutaneous Leishmaniasis Research Center, Faculty of Medicine, Mashhad University of Medical Sciences, Mashhad, Iran

Adv Dermatol Allergol 2019; XXXVI (3): 276-281
DOI: https://doi.org/10.5114/ada.2019.85639

\begin{abstract}
Introduction: The relationship between allergic and autoimmune diseases is an important issue, which has recently attracted the researchers' interest.

Aim: To determine the relationship between atopy and psoriasis.

Material and methods: This case-control study was conducted on 102 patients referred to the Ghaem Hospital, Mashhad, Iran, in 2016. The participants were assigned into two groups: experimental and control groups, including the patients suffering from psoriasis and those with no history of cutaneous or other systemic diseases, respectively. Both groups filled in the ISAAC questionnaire and had skin prick tests. In addition, the serum levels of immunoglobulin E (IgE) and blood eosinophil cell count were measured. The data were analysed using the regression test through SPSS version 16.

Results: According to the results of the ISAAC questionnaire, there was a significant difference between the control and experimental groups in terms of asthma $(p=0.04)$. The mean serum concentrations of IgE and eosinophil cell count were not significantly different between the experimental $(153.93 \mathrm{IU} / \mathrm{ml}$ and $187.77 \mathrm{cells} / \mu \mathrm{l}$, respectively) and control groups ( $152.19 \mathrm{IU} / \mathrm{ml}$ and 187.68 cells $/ \mu \mathrm{l}$, respectively) ( $p=0.057$ and $p=0.886$, respectively). In addition, there was an indirect correlation between the eosinophil cell count and psoriasis severity $(p=0.032, r=-0.297)$. Furthermore, the comparison of the skin prick test results revealed no significant difference between the two groups regarding the number of positive and negative cases $(p=0.436)$.

Conclusions: The findings suggested that atopy was not common in the patients with psoriasis and supported the concept that atopy protects against such autoimmune diseases such as psoriasis.
\end{abstract}

Key words: atopy, eosinophilia, immunoglobulin E, psoriasis.

\section{Introduction}

Psoriasis is an autoimmune chronic inflammatory disease, which is typically characterized by redness, itching, and scaly patches on the dermis. This skin condition is known as an idiopathic disease that may be induced due to abnormal T lymphocytes activity. Skin, nails, and joints are the most commonly involved areas in psoriasis [1]. Although the definitive aetiology and pathogenesis of this disease are still unknown, the environmental and genetic factors are proposed as the main causes of this disease. The positive family history in the patients with psoriasis has been reported in 35-90\% of cases [2].
The prevalence of psoriasis is reported to be $1-3 \%$, affecting 7.4 million adults in the United States [3]. This rate has remained stable since the mid-2000s. This disease can lead to remarkable distress and decrease the patient's quality of life [1]. The males and females are almost equally affected by psoriasis; furthermore, the children with involved parents are more prone to this disease than others [3]. The T-helper (Th) 1 and Th17 cells are responsible for the inflammation of psoriasis. However, Th2 cells have an antagonistic effect on the inflammation caused by these cells [2].

Besides, atopy refers to the genetic tendency to develop allergic diseases (e.g. eczema, allergic rhinitis, and

Address for correspondence: Maryam Khoshkhui, Allergy Research Center, Mashhad University of Medical Sciences, Ghaem Hospital, Shariati Square, Mashhad, Iran, phone: +98 51 38458769, fax: +98 51 38458769, e-mail: khoshkhuim@mums.ac.ir Received: 13.01.2018, accepted: 16.03.2018. 
asthma) [4] and produce immunoglobulin E (IgE) after the exposure to the allergens [5]. This disorder is typically associated with elevated immune responses to the common allergens, especially inhaled and food allergens [6]. A number of human diseases are induced by the immune responses to non-microbial environmental antigens involving the Th-cells producing interleukin (IL) 4, IL-5, and IL-13, IgE antibodies, such cells as mast cells, and eosinophils [7]. Recently, the association between autoimmune diseases and atopy has been considered as one of the important research issues. There are few studies investigating the relationship between atopy and psoriasis $[8,9]$. Nevertheless, the literature shows conflicting results on the association between psoriasis and lgE.

\section{Aim}

This study is aimed to determine the relationship between atopy and psoriasis. So, the history of the aforementioned allergic diseases was evaluated based on the International Study of Asthma and Allergies in Childhood (ISAAC). In addition, the serum levels of IgE and blood eosinophil cell count were measured, and the positive reaction in the skin prick test was considered as atopy.

\section{Material and methods}

This case-control study was conducted on the patients referred to the Department of Dermatology in Ghaem Hospital affiliated with the Mashhad University of Medical Sciences, Mashhad, Iran, in 2016. The participants were assigned into two groups: experimental and control groups, including the patients with psoriasis and subjects with no history of cutaneous or other systemic diseases.

\section{Study design}

A total of 102 participants (i.e., 52 and 50 cases in the experimental and control groups, respectively) were entered into the study using the purposive sampling technique. The age range of the participants was $18-75$ years. The exclusion criteria included pregnancy, cancer or inflammatory and other infectious diseases, and using immunosuppressive drugs.

The presence and severity of psoriasis were determined by a dermatologist, and the patients suspected of psoriasis were referred for a biopsy. All the patients were fully examined, and the severity of psoriasis was estimated using the Psoriasis Area and Severity Index (PASI), which reflects body surface involvement, erythema, scaling, and induration in each region.

All obtained results were recorded in a researchermade checklist. Additionally, the patients were referred to an allergy specialist. After providing the participants with a complete explanation and obtaining their agreement, they were referred to the Ghaem Clinical Labora- tory for blood sampling and evaluation of eosinophil count and IgE level. The serum IgE and the complete blood count analysis were estimated using the enzymelinked immunosorbent assay in Sysmex apparatus (EUROIMMUNE Inc., Germany). Additionally, the blood smear samples were stained with Giemsa staining.

Subsequently, all the patients were investigated for the history of allergies, such as asthma and allergic rhinitis, based on the ISAAC questionnaire. Then, the skin prick test was performed to determine the sensitivity to five common allergens [10] and the obtained results were noted in the patients' medical records.

\section{Ethical consideration}

In accordance with the ethical principles, an approval was obtained from the Ethics Committee of the Mashhad University of Medical Sciences. Furthermore, the informed consent was obtained from all the participants. Additionally, they were assured about the confidentiality of their information and the possibility of withdrawal from the study at any stage.

\section{Statistical analysis}

Data were analysed using the regression test through SPSS software version 16. A p-value less than 0.005 was considered statistically significant.

\section{Results}

According to the results of the study, $63.5 \%$ and $68 \%$ of the participants in the experimental and control groups, respectively, were female. Therefore, the two groups were comparable in terms of gender $(p=0.62)$. In addition, the mean ages of the patients in the experimental and control groups were $38.46 \pm 14.74$ and 36.54 \pm 15.34 years, respectively. There was no significant difference between the two group regarding the age ( $p=$ 0.52). In the control group, the mean ages of the subjects with positive and negative skin prick test results were $36 \pm 14.44$ and $37.08 \pm 16.48$ years, respectively. Similarly to the experimental group, there was no significant difference between the skin prick test results regarding the age in the control group $(p=0.806)$.

The mean duration of psoriasis was $8.08 \pm 8.58$ months in the experimental group. The frequencies of the types of allergies and psoriasis are shown in Table 1. Figure 1 displays the frequency of allergic rhinitis in different seasons.

The mean PASI score was $11.84 \pm 6.52$ in the experimental group. Furthermore, the mean ages of the subjects in the experimental group were $35.36 \pm 13.93$ and $40.73 \pm 15.13$ years, respectively.

As the results indicated, $42.3 \%$ and $57.7 \%$ of the patients in the experimental group had positive and negative results in the skin prick test, respectively. Fur- 
Table 1. The frequency of the allergies and psoriasis types

\begin{tabular}{lcccc}
\hline Variables & Types & Control group & Experimental group & $P$-value \\
\hline Allergies & Asthma & 12 & 1.9 & 0.044 \\
\cline { 2 - 5 } & Eczema & 26 & 26.9 & 0.916 \\
\cline { 2 - 5 } & Allergic rhinitis & 34 & 23.07 & 0.221 \\
\hline Psoriasis & Plaque psoriasis & 0 & 69.2 & - \\
\cline { 2 - 5 } & Guttate & 0 & 5.8 & - \\
\cline { 2 - 5 } & Inverse & 0 & 11.5 & - \\
\cline { 2 - 5 } & Pustular & 0 & 5.8 & - \\
\hline
\end{tabular}

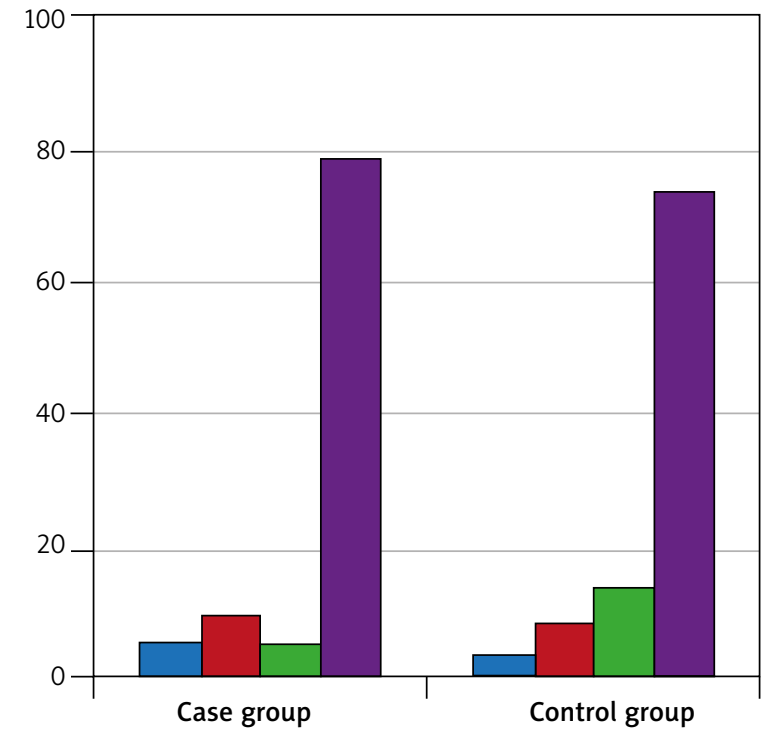

Late winter to early spring $\quad$ Late summer to early fall Total

Figure 1. The frequency of allergic rhinitis in different seasons

thermore, the rates of positive and negative results were equal in the control group (i.e., $50 \%$ positive and $50 \%$ negative). The comparison of the skin prick test results showed no significant difference between the two groups regarding the number of positive and negative cases $(p=0.436)$.

The study of the frequency of skin prick test results in the experimental group based on gender revealed that $55.5 \%$ of the patients with a positive reaction and $70 \%$ of the subjects with a negative reaction were female. In addition, in the control group, $60 \%$ of the patients with a positive reaction and $76 \%$ of the subjects with a negative reaction were female. The results demonstrated no significant difference between the two groups considering the prevalence of atopic dermatitis $(p=0.253)$.
The frequency of the sensitivity to allergens is illustrated in Table 2. The comparison of the prevalence of sensitivity to the common allergens between the study groups showed no significant difference between the two groups in this regard $(p>0.005)$. The comparison of skin prick test results in the experimental group revealed no significant difference between the patients with positive and negative results in terms of the age $(p=0.19)$.

The means of IgE level and eosinophil counts were $153.08 \pm 217.18 \mathrm{lU} / \mathrm{ml}$ and $187 \pm 116.04$ cells $/ \mu \mathrm{l}$, respectively. The distribution of eosinophil count was abnormal in both experimental and control groups ( $p=0.025$ and $p=0.189$, respectively). The means of eosinophil count were $187.77 \pm 129.43$ and $187.68 \pm 101.57$ cells/ $\mu$ in the experimental and control groups, respectively. Furthermore, there was no significant difference between the two groups in this regard $(p=0.886)$.

Furthermore, the eosinophil count and PASI scores had an indirect correlation $(r=-0.29, p=0.032)$. Likewise, the results of the regression test revealed a negative causal relationship between these two variables $\left(R^{2}=0.07, p<0.05\right)$, according to which about $7 \%$ of the eosinophil count variations were related to the PASI levels. The comparison of the eosinophil count with its normal range (i.e., 450 cells/ $\mu$ l) showed that there was a significant difference between these two variables in the patients $(p<0.005)$. Furthermore, no significant correlation was observed between the eosinophil count and psoriasis duration in the experimental group $(r=-0.009$, $p>0.05$ ).

According to the results, $3.84 \%$ and $96.16 \%$ of the patients had positive and negative eosinophil counts, respectively. Regarding the control group, these rates were $2 \%$ and $98 \%$, respectively. The two groups were comparable in terms of the eosinophil count $(p=0.58)$. The means of IgE level were $153.93 \pm 254.94$ (range: $0.8-1244$ IU/ $\mathrm{ml}$ ) and $152.19 \pm 171.97 \mathrm{IU} / \mathrm{ml}$ (range: $3-754 \mathrm{kU} / \mathrm{l}$ ) in the experimental and control groups, respectively. The distribution of IgE level was abnormal in both experimental and control groups ( $p=0.005$ and $p=0.004$, respec- 
tively). In addition, the two groups showed no significant difference in this regard ( $p=0.057)$.

Furthermore, there was no significant difference between the lgE level and its normal range $(150 \mathrm{lU} / \mathrm{ml})$ in the participants $(p=0.886)$. The results indicated no significant relationship between the IgE and PASI levels in the experimental group $(r=1, p=0.435)$. In addition, there was no significant relationship between the IgE level and psoriasis duration in the experimental group $(r=-0.042, p>0.005)$. In the experimental group, $28.84 \%$ and $71.16 \%$ of the patients had the IgE levels of $>150$ and $<150 \mathrm{IU} / \mathrm{ml}$, respectively. Accordingly, the IgE levels were high and low in $30 \%$ and $70 \%$ of the subjects. However, the results revealed no significant difference between the two groups in this regard $(p=0.89)$.

\section{Discussion}

The relationship between autoimmune diseases and atopy is a subject that has recently attracted the researchers' interests. It is hypothesized that the diseases caused by the reciprocal counter-regulation of type-1 and type-2 pathogenic mechanisms might not happen or would be less severe for the same individual. The fact that the signature cytokines and transcription factors are both mandatory and adequate for polarization in one direction or the other prove the presence of the Th1/Th2 parameters [11].

Some clinical and histopathological findings like some types of atopy are caused by psoriasis [12, 13]. Th17 and Th1 cells are the determining factors of inflammation in both diseases. In this regard, Th1 has a pathogenic role in atopic diseases, such as atopic dermatitis [14]. In this study, we aimed to find whether the presence of atopy is common in the patients with psoriasis or not.

As the findings of the present study indicated, based on the ISAAC questionnaire, no significant difference was observed between the patients with psoriasis and atopy regarding the type of atopy, except for asthma. Our results revealed that the control group had a higher rate of asthma, compared to the experimental group.

In a study conducted by Hajdarbegovic et al., the possibility of asthma was reported to be lower in the patients with psoriasis vulgaris; however, the rate of allergic rhinitis was not different between the two groups [4]. Similar to the mentioned study, the results of our study showed that the rate of asthma was lower in the patients with psoriasis; however, we did not isolate different types of psoriasis. Therefore, it is suggested to conduct further studies to assess the relationship between atopy and psoriasis. In addition, more studies are needed to investigate the frequencies of asthma and allergic rhinitis in the different types of psoriasis.

In another study, atopic disorders which were assessed by the ISAAC questionnaire had a higher prevalence in the patients with autoimmune disease than that
Table 2. The frequency of sensitivity to allergens

\begin{tabular}{lccc}
\hline Allergen & $\begin{array}{c}\text { Positive/ } \\
\text { negative }\end{array}$ & $\begin{array}{c}\text { Experimental } \\
\text { group }\end{array}$ & $\begin{array}{c}\text { Control } \\
\text { group }\end{array}$ \\
\hline Salsola & Positive & 34.6 & 36 \\
\cline { 2 - 4 } & Negative & 65.4 & 64 \\
\hline Pigweed & Positive & 11.5 & 12 \\
\cline { 2 - 4 } & Negative & 88.5 & 88 \\
\hline Grass mix & Positive & 7.7 & 16 \\
\cline { 2 - 4 } & Negative & 92.3 & 84 \\
\hline Tree mix & Positive & 15.4 & 18 \\
\cline { 2 - 4 } & Negative & 84.6 & 82 \\
\hline D. farinae & Positive & 13.5 & 26 \\
\cline { 2 - 4 } & Negative & 86.5 & 74 \\
\hline
\end{tabular}

in the control group. However, there was no difference between the two groups regarding the development of asthma [15]. In the mentioned study, the frequency of asthma was higher in the patients with autoimmune diseases than in other subjects. This inconsistency with our study can originate from the difference in the type of autoimmune disease which was evaluated [15].

As we know, psoriasis is a part of an autoimmune spectrum in which diverse diseases share common underlying genetic susceptibility loci and treatment modalities. Atopy is caused by type I hypersensitivity to specific antigens through impacts of an antibody test [8]. Atopy diagnosis is performed differently in various studies. In this regard, while some studies rely on a positive prick test for atopy detection, other studies use a high level of IgE as the main determinant of this disease, and still others base the atopy diagnosis on the ISAAC questionnaire [11].

In this study, based on the results of the skin prick test, no significant difference was observed between the two groups in terms of atopy. There are few studies investigating the atopy in psoriasis featuring specific IgE. There is only one similar study utilizing the skin prick test to assess the association between atopy and psoriasis [8]. In line with our study, Ünal et al. demonstrated that the sensitivity to mites detected by skin prick tests were increased in the psoriatic patients. Also, it was indicated that the atopy history and total IgE level were higher in the patients with psoriasis compared to those in the healthy subjects [16].

Nonetheless, some studies reported elevated total IgE levels in the psoriatic patients [17]. This difference might be due to the difference in disease severity and the employment of various laboratory techniques. In a study conducted by Ovcina-Kurtovic et al., the total serum IgE level was measured using the nephelometric method. They reported significantly higher total serum levels of 
IgE in the patients with psoriasis, compared to that in the controls $[17,18]$.

Our results were inconsistent with Li et al. findings in 2005, which compared erythrodermic psoriasis with psoriasis vulgaris; they indicated that the change in Th2 cell phenotype was introduced as the cause of high IgE levels in erythrodermic psoriasis [19]. It is also not similar to other studies that showed the IgE level was higher in the erythrodermic and plaque psoriasis patients than in the control group [17].

Since Th1 is the dominant phenotype of Th cells in psoriasis vulgaris, IgE level is lower in the patients with psoriasis vulgaris patients than in the erythrodermic psoriasis patients. Nevertheless, this discrepancy could be due to the fact that Li et al. investigated the erythrodermic psoriasis patients and used no control group. It was possible to obtain similar findings in case we employed a larger sample size and isolated the types of psoriasis.

Similar studies have highlighted the role of IgE level in other autoimmune diseases [8, 20, 21]. However, no relationship was observed between the levels of IgE and psoriasis in our study. This discrepancy between our results and those of the aforementioned studies could be due to not separating different types of psoriasis. It was possible to obtain comparable results if the subjects were separated in terms of the type of psoriasis.

As the findings of the present study indicated, there was no difference between the experimental and control groups in terms of the eosinophil count. Furthermore, an indirect correlation was observed between the eosinophil count and PASI level, which was in line with the results reported by similar studies [22].

Ansarin et al. underscored the main role of Th1 cytokine profiles in the pathogenesis of psoriasis. They demonstrated eosinophil count reduced with the enhancement of PASI level [23]. Furthermore, Michaëlsson et al. reported no significant difference between the patients with psoriasis and control group in terms of the eosinophilic cationic protein level [24]. Since tumour necrosis factor- $\alpha$ is the main cytokine produced by Th1 and Th17 in psoriasis, it can inhibit the switching of Th1 cell cytokine profile to the Th2 cells. The cause of low eosinophil count in these patients is the function of Th17 and Th1 cells [16].

Inconsistent with this study, in a study conducted by Mansur et al., the number of eosinophil count was higher in the patients with psoriasis, compared to that in the basal cell carcinoma patients [25]. Nevertheless, the mentioned study did not employ a healthy control group and had a smaller sample size, compared to our study. The present study revealed the role of eosinophil as an independent factor in the pathogenesis of psoriasis. However, this cell was found to be an ineffective factor in the diagnosis of atopy. In our study, no difference was observed between the experimental and control groups in terms of the eosinophil count.
The limitations of the present study included the small sample size and lack of enough time for completing the questionnaires for all of the patients. Further studies are recommended to evaluate the pathogenesis of the diseases, soluble LAG-3, and CD30 level. It is also suggested that T cell phenotype in the patients with psoriasis and allergic disorders be compared with normal subjects.

\section{Conclusions}

Based on the results of the skin prick test, there was no significant difference between the two groups in terms of atopy. This test indicated that the prevalence of asthma was lower in the patients with psoriasis. Furthermore, the results of the ISAAC questionnaire revealed no significant difference between the patients with psoriasis and atopy regarding the type of atopy, except for asthma. Consequently, it is possible that psoriasis and asthma have protective effects against each other. These findings suggested that atopy was not common in psoriasis patients and supported the concept that atopy may protect against autoimmune diseases such as psoriasis.

\section{Acknowledgments}

Hereby, we extend our gratitude to the Deputy of Research and Technology as well as all members and experts of the Research Council of the Mashhad University of Medical Sciences for their approval of the project proposal. We wish to thank Dr. Mohsen Tizro and Dr. Nasrin Motevali for helping us with the prick test. We also wish to thank all the patients and colleagues for their cooperation in the present study.

\section{Conflict of interest}

The authors declare no conflict of interest.

\section{References}

1. Lee MR, Cooper AJ. Immunopathogenesis of psoriasis. Austral J Dermatol 2006; 47: 151-9.

2. Steinman L. A brief history of Th17, the first major revision in the Th1/Th2 hypothesis of T cell-mediated tissue damage. Nat Med 2007; 13: 139-45.

3. Rachakonda TD, Schupp CW, Armstrong AW. Psoriasis prevalence among adults in the United States. J Am Acad Dermatol 2014; 70: 512-6.

4. Hajdarbegovic E, Nijsten T, Westgeest A, et al. Decreased prevalence of atopic features in patients with psoriatic arthritis, but not in psoriasis vulgaris. J Am Acad Dermatol 2013; 68: 270-7.

5. Borish L. Allergic rhinitis: systemic inflammation and implications for management. J Allergy Clin Immunol 2003; 112: 1021-31.

6. Sabat R, Philipp S, Höflich C, et al. Immunopathogenesis of psoriasis. Exp Dermatol 2007; 16: 779-98. 
7. Khoshkhui M, Alyasin S, Sarvestani EK, et al. Evaluation of serum interleukin-35 level in children with persistent asthma. Asian Pac J Allergy Immunol 2017; 35: 91-5.

8. Ünal ES, Gul U, Dursun AB, Erkekol FO. Prediction of atopy via total immunoglobulin E levels and skin prick tests in patients with psoriasis. Turk J Med Sci 2017; 47: 577-82.

9. Eyerich S, Onken AT, Weidinger S, et al. Mutual antagonism of T cells causing psoriasis and atopic eczema. N Engl J Med 2011; 365: 231-8.

10. Oskouei YM, Hosseini RF, Ahanchian H, et al. Report of common aeroallergens among allergic patients in northeastern Iran. Iran J Otorhinolaryngol 2017; 29: 89-94.

11. Rabin R, Levinson A. The nexus between atopic disease and autoimmunity: a review of the epidemiological and mechanistic literature. Clin Exp Immunol 2008; 153: 19-30.

12. Schleicher SM. Psoriasis: pathogenesis, assessment, and therapeutic update. Clin Podiatr Med Surg 2016; 33: 355-66.

13. Kneilling M, Röcken M. Mast cells: novel clinical perspectives from recent insights. Exp Dermatol 2009; 18: 488-96.

14. Hueber AJ, McInnes IB. Immune regulation in psoriasis and psoriatic arthritis - recent developments. Immunol Letters 2007; 114: 59-65.

15. Rudwaleit M, Andermann B, Alten R, et al. Atopic disorders in ankylosing spondylitis and rheumatoid arthritis. Ann Rheum Dis 2002; 61: 968-74.

16. Malisiewicz B, Murer C, Pachlopnik Schmid J, et al. Eosinophilia during psoriasis treatment with TNF antagonists. Dermatology 2011; 223: 311-5.

17. Ovcina-Kurtovic N, Kasumagic-Halilovic E. Serum levels of total immunoglobulin $\mathrm{E}$ in patients with psoriasis: relationship with clinical type of disease. Med Arh 2010; 64: 28-9.

18. Przybilla B, Ring J, Volk M. Total IgE levels in the serum in dermatologic diseases. Hautarzt 1986; 37: 77-82.

19. Li LF, Sujan SA, Yang H, et al. Serum immunoglobulins in psoriatic erythroderma. Clin Exp Dermatol 2005; 30: 125-7.

20. Adkinson Jr NF, Bochner BS, Burks AW, et al. Middleton's Allergy: Principles and Practice. Elsevier Health Sciences 2013.

21. Karimi P, Modarresi SZ, Sahraian MA, et al. The relation of multiple sclerosis with allergy and atopy: a case control study. Iran J Allergy Asthma Immunol 2013; 12: 182-9.

22. Jullien D, Barker J. Genetics of psoriasis. J Eur Acad Dermatol Venereol 2006; 20 (Suppl 2): 42-51.

23. Ansarin H, Shekarabi M, Behrangi E. Investigation of the relationship between serum levels of cytokines secreted from Th1 and Th2 cells, IL8 and ICAM-1 and psoriasis severity based on PASI. Razi J Med Sci 2010; 16: 32-9.

24. Michaëlsson G, Kraaz W, Gerdén B, et al. Patients with psoriasis have elevated levels of serum eosinophil cationic protein and increased numbers of EG2 positive eosinophils in the duodenal stroma. Br J Dermatol 1996; 135: 371-8.

25. Mansur A, Göktay F, Yaşar Ş. Peripheral blood eosinophilia in association with generalized pustular and erythrodermic psoriasis. J Eur Acad Dermatol Venereol 2008; 22: 451-5. 